\title{
Regulatory Mechanisms and Promising Applications of Quorum Sensing-Inhibiting Agents in Control of Bacterial Biofilm Formation
}

OPEN ACCESS

Edited by:

Rodolfo García-Contreras,

National Autonomous University

of Mexico, Mexico

Reviewed by:

Kibaek Lee,

Chonnam National University,

South Korea

Fohad Mabood Husain,

King Saud University, Saudi Arabia

Fazlurrahman Khan,

Sharda University, India

${ }^{*}$ Correspondence:

Jianyi Pan

jianyi.pan@zstu.edu.cn

Specialty section:

This article was submitted to Antimicrobials, Resistance

and Chemotherapy,

a section of the journal

Frontiers in Microbiology

Received: 31 July 2020 Accepted: 23 September 2020

Published: 15 October 2020

Citation:

Zhou L, Zhang Y, Ge Y, Zhu X and Pan J (2020) Regulatory Mechanisms

and Promising Applications

of Quorum Sensing-Inhibiting Agents

in Control of Bacterial Biofilm

Formation.

Front. Microbiol. 11:589640.

doi: 10.3389/fmicb.2020.589640

\section{Lantian Zhou, Yue Zhang, Yongze Ge, Xuan Zhu and Jianyi Pan*}

Zhejiang Provincial Key Laboratory of Silkworm Bioreactor and Biomedicine, College of Life Sciences and Medicine,

Zhejiang Sci-Tech University, Hangzhou, China

A biofilm is an assemblage of microbial cells attached to a surface and encapsulated in an extracellular polymeric substance (EPS) matrix. The formation of a biofilm is one of the important mechanisms of bacterial resistance, which not only leads to hard-tocontrol bacterial infections in humans and animals but also enables bacteria to be a major problem in various fields, such as food processing, wastewater treatment and metalworking. Quorum sensing (QS) is a bacterial cell-to-cell communication process that depends on the bacterial population density and is mediated by small diffusible signaling molecules called autoinducers (Als). Bacteria use QS to regulate diverse arrays of functions, including virulence and biofilm formation. Therefore, the interference with QS by using QS inhibiting agents, including QS inhibitors (QSIs) and quorum quenching (QQ) enzymes, to reduce or even completely repress the biofilm formation of pathogenic bacteria appears to be a promising approach to control bacterial infections. In this review, we summarize the mechanisms of QS-regulating biofilm formation and QSinhibiting agents that control bacterial biofilm formation, strategies for the discovery of new QS inhibiting agents, and the current applications of QS-inhibiting agents in several fields to provide insight into the development of effective drugs to control pathogenic bacteria.

Keywords: biofilm formation, quorum sensing, QS inhibitors, quorum quenching, bacteria

\section{INTRODUCTION}

A biofilm is a large number of bacterial cell aggregates coated in an extracellular mucous comprised of a polysaccharide matrix, lipids, and proteins, which they secrete (Sutherland, 2001; Branda et al., 2005). Bacteria tend to form biofilms when exposed to external environmental pressure, such as extreme nutrient deficiency or excess, high osmotic pressure, low $\mathrm{pH}$, oxidative stress, antibiotics and antimicrobial agents (Costerton et al., 1994). It is a state of self-protection formed when bacteria grow on the surfaces of objects under natural conditions. Any bacteria in nature can form a biofilm under mature conditions, and more than $90 \%$ of bacteria live and grow primarily in the form of biofilms (Donlan, 2002).

Biofilms may irreversibly form on a wide variety of surfaces, including living tissues, industrial or potable water system piping, indwelling medical devices, and natural aquatic systems, and once 
it is formed, it cannot be removed by gentle rinsing (Donlan, 2002). More than $60 \%$ of all bacterial infections are caused by biofilm formation, according to a public announcement from the National Institutes of Health (NIH) (Lewis, 2001). The formation of biofilms leads to not only common bacterial infections, such as infections of the urinary tract, catheters, children's middleear, common dental plaque formation, and gingivitis, but also to hard-to-treat or relapsing infections and severe infections that cause serious morbidity and mortality (Lewis, 2001).

Currently, the use of antibiotics is still the major treatment for bacterial infectious diseases. However, biofilms, being a barrier that exists around bacterial cells, reduces the susceptibility of bacteria to antibiotics and causes persistent infections (Stewart, 2002). It has been shown that bacteria in a biofilm increase their resistance against antibiotics by about 1000-fold (Hoiby et al., 2010). Thus, it is hard to control bacterial infections with conventional antibiotics due to the presence of biofilms. Therefore, it is urgent to find a strategy to inhibit the formation of biofilms to control these increasingly serious infections.

At present, it is well known that bacteria forming a biofilm is under the control of the quorum sensing system (QS) (Ding et al., 2011). The QS involves cell-to-cell communication among bacteria using small diffusible chemical signaling molecules called autoinducers (AIs) (Waters and Bassler, 2005). The signaling molecules accumulate in the surrounding environment with an increase of bacterial density. When the concentration of signaling molecules reaches a minimal threshold, they bind to receptor proteins, thereby activating the expression of genes associated with biofilm formation (Williams, 2007). QS inhibiting agents, including QS inhibitors (QSIs) and quorum quenching (QQ) enzymes, can cut off QS cell communication via a variety of mechanisms, consequently inhibiting the formation of biofilms (Augustine et al., 2010; Chatterjee et al., 2017). In addition, QS inhibiting agents can also increase bacterial sensitivity to antibiotics (Ozcan et al., 2019). Therefore, the use of QS inhibiting agents would be a promising approach to control bacterial infections.

In this review, we summarize the mechanisms by which QS inhibiting agents regulate biofilm formation, some strategies for the discovery of new QS inhibiting agents, and the current applications of QS inhibiting agents in medical and industrial fields. We aim to provide a new perspective for exploring more effective antibiotic drugs through the use of QS inhibiting agents.

\section{MECHANISMS OF BIOFILM FORMATION REGULATED BY QS}

Quorum sensing regulatory networks are not only very complicated but also vary among bacterial species (Solano et al., 2014). Therefore, the regulatory mechanisms of QS on biofilm formation cannot be described in general. However, based on the types of employed AIs, QS systems can be divided into several categories, namely, AHL system, AIP system (these two system known as AI-1 system previously), AI-2 system and AI-3 system.

The AHL system exists in Gram-negative bacteria and the signaling molecules employed in this system are $\mathrm{N}$-acyl homoserine lactones (AHLs) (Slock et al., 1990; Stevens et al., 1994). While, autoinducing peptides (AIPs) are employed in the AIP system and this system is found only in Grampositive bacteria (Kleerebezem et al., 1997; Irie and Parsek, 2008). Presently, there are a large number of studies on these two systems.

However, there are only a few reports about AI-2 and AI3 systems, although these two systems have been found to be present in both Gram-positive and Gram-negative bacterial species and to participate in interspecies signal exchange (Surette and Bassler, 1998). AI-2 signaling molecules are a class of furanosyl borate diesters whose precursors are 4,5-dihydroxy2,3-glutara dione (DPD) (Bassler et al., 1997). The AI-3 signaling molecules have recently been identified as pyrethroids (Sperandio et al., 2003; Kim et al., 2020). The mechanism of how the QS system regulates biofilm formation is described in detail below for Gram-negative and positive bacteria separately.

\section{QS Regulation of Biofilm Formation in Gram-Negative Bacteria}

The signaling molecule of the AHL system in Gram-negative bacteria is AHL. The AHL-mediated QS system was first found in Vibrio fischeri (Nealson and Hastings, 1979). In this bacterium, the AHL signal, $N$-(3-oxohexanoyl)-L-homoserine lactone (OHHL), is biosynthesized by AI synthase LuxI, and the resulting OHHL diffuses out of the bacterial cell. When the concentration of OHHL reaches a critical threshold with the increase of cell density, OHHL binds to LuxR, which is not only an OHHL receptor, but also a DNA-binding transcriptional activator, thereby activating the expression of genes associated with biofilm formation (Engebrecht et al., 1983; Engebrecht and Silverman, 1984). Presently, it is well known that this regulatory process is a typical model for the regulation of biofilm formation by AHL systems in most Gram-negative bacteria.

Take Pseudomonas aeruginosa for instance, which has two AI synthase genes, lasI and rhlI, which both share significant sequence homologies to luxI of $V$. fischeri (Lee and Zhang, 2015). Their signals, $N$-(3-oxo-dodecanoyl)-lhomoserine lactone (OdDHL) and $N$-butyryl-l-homoserine lactone (BHL), are separately synthesized by LasI and RhlI. When they reach the concentration threshold, these two AHL signaling molecules bind to their receptors, LasR and RhlR, respectively, to activate the expression of regulatory genes related to biofilm formation and virulence (Wade et al., 2005). Among these two AHL systems, the $r h l$ system is involved in regulating swarming motility that participates in the early stage of biofilm establishment (Khan et al., 2020c), and the biosynthesis of virulence factors, such as rhamnolipid and pyocyanine (Winzer et al., 2000; Daniels et al., 2004; Dusane et al., 2010). The las system controls genes encoding elastase, alkaline protease, endotoxin $\mathrm{A}$ and other genes related to biofilm formation (Wilder et al., 2011).

In addition, in $P$. aeruginosa, there are also two other types of AHLs-mediated systems, the pqs system and the iqs system. These two systems work in a way similar to the $r h l$ and las systems, though their AIs, PQS (2-heptyl3-hydroxy-4-quinolone) and IQS 
(2-(2-hydroxyphenyl)-thiazole-4-carbaldehyde), are chemically different from AHLs (Lee and Zhang, 2015). Additionally, the pqs system has been reported to be related to the synthesis of bacterial extracellular DNA, which is important for the formation of biofilms (Allesen-Holm et al., 2006). In brief, the four AHL systems, las, rhl, iqs and $p q s$, cross-interact to form a complicated QS network that co-regulates biofilm formation by $P$. aeruginosa.

The AHL system is also involved in the regulation of biofilm formation in Escherichia coli (Walters and Sperandio, 2006). However, different from the AHL system in $P$. aeruginosa, only the receptor gene sdiA homologous to $l u x R$ is found and the AHL synthase gene homologous to $l u x I$ is absent (Walters and Sperandio, 2006). Hence, it is speculated that the receptor SdiA may respond to the AHLs produced by other bacterial species to regulate biofilm-related gene expression. The finding that in the presence of exogenous AHLs, there is an increase of EPS production in E. coli and the attachment of bacterial cells (Aswathanarayan and Vittal, 2016) confirms that bacteria can utilize the signaling molecules of other bacterial species for biofilm formation.

Currently, AI-2 systems have been found to affect biofilm formation in several Gram-negative species, such as Helicobacter pylori, E. coli, V. parahemolyticus, P. aeruginosa and V. cholerae (Hammer and Bassler, 2003; Li et al., 2007; Anderson et al., 2015; Li et al., 2015; Guo et al., 2018). However, at present, only the regulatory mechanisms of the AI-2 systems in E. coli and $V$. cholerae have been clarified. In E. coli, the AI-2 signaling molecule is transported into the cell by an $\mathrm{ABC}$ transporter protein when the extracellular concentration of the AI-2 signaling molecule reaches its threshold (Li et al., 2007). The resulting signaling molecule is then phosphorylated by LsrK kinase followed by binding to the transcriptional regulator LsrR, thereby activating gene expression ( $\mathrm{Li}$ et al., 2007). In V. cholerae, the AI2 signaling molecule is detected by the receptor complex LuxPQ. When the signaling molecule concentration reaches its threshold, the kinase activity of LuxQ is converted to phosphatase, which dephosphorylates downstream regulatory protein LuxO, resulting in the production of transcriptional regulatory protein HapR, thereby inhibiting the transcription of genes related to biofilm formation (Hammer and Bassler, 2003, 2007).

AI-3 signaling molecules are currently known to be related to the formation of flagellum and adhesin in E. coli (Sperandio et al., 2002), but their regulatory mechanisms for biofilm formation remain unclear.

\section{QS Regulation of Biofilm Formation in Gram-Positive Bacteria}

In Gram-positive bacteria, the AIs of the AIP system are AIPs, and the regulation of biofilm formation by the AIPs-mediated QS system is also a typical pattern. Bacteria produce a small oligopeptide in their cells, and the oligopeptide is processed into a mature AIP through modification and then it is transported outside of the cells (Sturme et al., 2002). When the concentration of AIP reaches its threshold, it binds to the extracellular segment of histidine kinase, a transmembrane receptor localized on the cell membrane, which leads to the activation of the kinase, followed by phosphorylation of downstream response regulatory factors, resulting in regulation of the expression of genes related to biofilm formation (Sturme et al., 2002).

For example, in Staphylococcus aureus, five genes, agrA, $\operatorname{agr} B$, agrC, agrD and hld in the agr operon constitute the agr AIP system (Painter et al., 2014). The signaling AIP is converted from its precursor peptide $\operatorname{AgrD}$, and $\operatorname{AgrB}$, a transmembrane protein, is responsible for the conversion of AgrD to mature AIP and transportation of the resulting AIP outside of the cell. When the extracellular AIP concentration reaches its threshold, the AIP binds to an extracellular part of AgrC, an integral transmembrane protein functioning as a histidine kinase, resulting in the activation of the kinase. The activated AgrC in turn phosphorylates the downstream response regulator AgrA. Phosphorylated AgrA binds to the intergenic DNA between promoters $\mathrm{P} 2$ and P3, and thus activates promoter transcription. The agr system has been shown to play a major role in the dispersion phase, which is the last stage of biofilm formation (Yarwood et al., 2004). Agr mutants formed a thicker biofilm comparing with the wild type, but this increased biofilm thickness has been attributed to the inability of cells to detach from the mature biofilm, not to cell growth or death (Vuong et al., 2000, 2004).

Presently, there are several reports about the regulation of biofilm formation by the AI-2 system in Gram-positive bacteria. For example, a study has shown that the lack of $\operatorname{luxS}$, which encodes AI-2 synthase, promotes the transcription of $r b f$, a positive regulatory factor for biofilm formation, and results in an increase of biofilm formation and higher levels of production of polysaccharide intercellular adhesion (PIA) in S. aureus (Ma et al., 2017). However, the opposite finding, that the absence of luxS decreases biofilm formation, was also found in Enterococcus faecalis (Wang et al., 2011; Yang et al., 2018) and Streptococcus suis (Wang et al., 2011; Yang et al., 2018). Nevertheless, the AI2 system is involved in the regulation of biofilm formation in Gram-positive bacteria, but its regulatory mechanism has not yet been fully characterized.

In addition, there is still no report about the AI-3 system in regard to the control of biofilm formation in Grampositive bacteria.

\section{THE MECHANISMS OF QS INHIBITING AGENTS SUPPRESSING BIOFILM FORMATION}

In the past two decades, a number of effective QS inhibiting agents have been developed and successfully used to control the formation of bacterial biofilms. These QS inhibiting agents are mainly QS inhibitors (QSIs) and quorum quenching (QQ) enzymes. Although different types of QSIs or QQ enzymes interfere with different parts of the QS signaling pathway, the inhibitory mechanism can be divided into three categories based on their functional targets, i.e., targeted to AI signaling molecules, receptors and downstream signaling cascades (Figure 1). These three mechanisms are described in detail with examples as follows. 

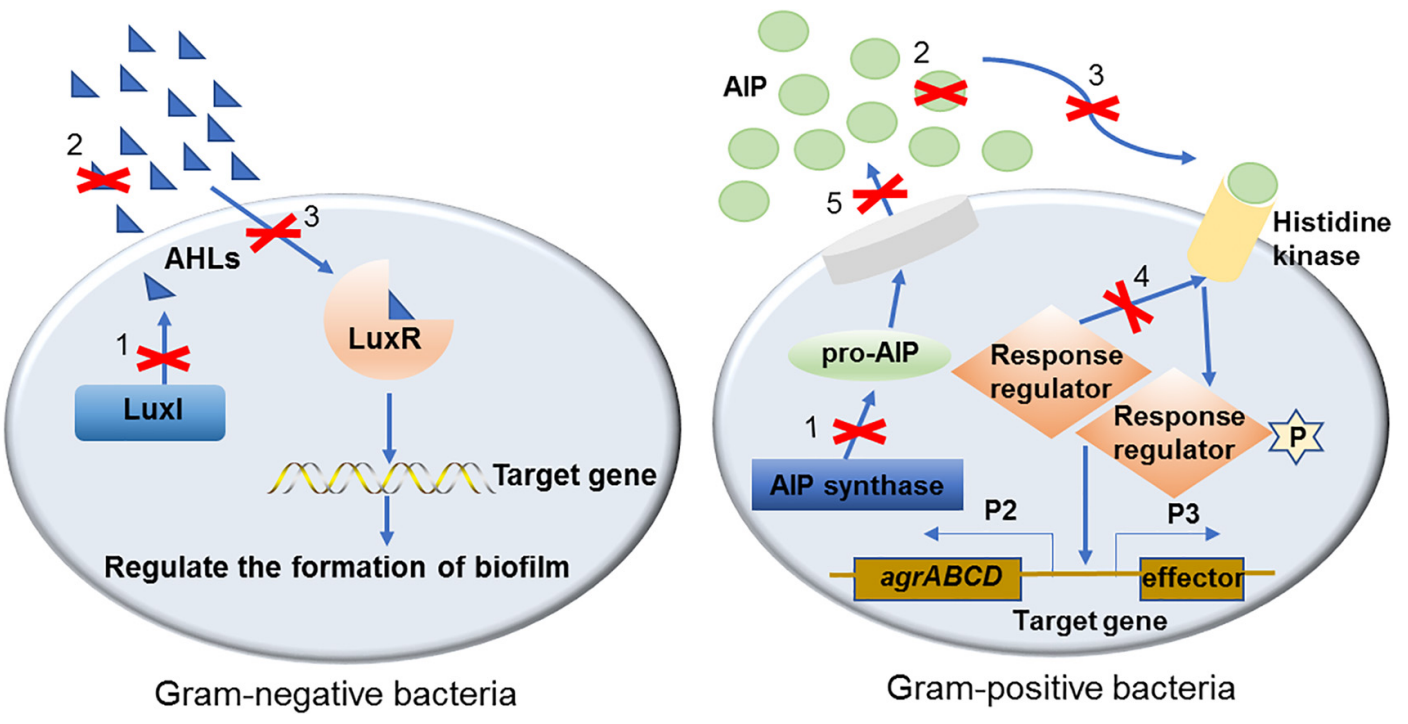

FIGURE 1 | The mechanisms of QS inhibiting agents in controlling bacterial biofilm formation. Mechanisms of QS inhibiting agents in controlling bacterial biofilm formation are marked with numbers on the diagram: (1). Inhibit Als synthesis; (2). Degrade or inactivate Als by AHL-lactonases, oxidoreductases, antibodies, etc.; (3). Interfere with the signal receptors using Al antagonists; (4). Interfere with the response regulators thus disturbing signaling cascade; (5). Reduce the extracellular Als accumulation by inhibiting Als efflux hence inhibited cell-to-cell signaling.

\section{Target Signal Molecule}

The QS inhibiting agents that target the AI signaling molecules are mainly AHL-lactonases, oxidoreductases, antibodies, and some other molecular compounds (Table 1). These agents inhibit the QS system by inactivation of signaling molecule synthases, neutralization of AIPs with antibodies, modification or degradation of the signaling molecules, etc.

Degradation or neutralization of QS signaling molecules by QQ enzymes are a direct and effective way to inhibit the QS system. In Gram-negative bacteria, AHLs can be degraded by two types of hydrolases, AHL-lactonase and AHL-acylase. An AHL-lactonase, encoded by aiiA of Bacillus spp and belonging to the metallo- $\beta$-lactamase superfamily, has been determined to effectively inhibit biofilm formation and attenuate virulence factors in several bacterial species (Augustine et al., 2010; dos Reis Ponce et al., 2012; Anandan and Vittal, 2019). Another QQ enzyme, AHL-acylase, is not widespread in Gram-negative bacteria. However, the enzyme has been found to be present at least in $P$. aeruginosa, and it has been shown to degrade AHLs with side chains and hence enable the bacteria to modulate the QS system (Sio et al., 2006). Currently, there are few reports on the degradation of AIPs by QQ enzymes in Gram-positive bacteria. However, AIPs can be neutralized by antibodies and cause interruption of QS signaling. For example, an anti-AI monoclonal antibody can efficiently inhibit QS via neutralization of an AI peptide (AIP-4) that is produced by S. aureus (Park et al., 2007).

$N$-acyl homoserine lactones oxidoreductase, another class of QQ enzyme, has also been reported in Gram-negative bacteria, it modifies the AIs and thereby attenuates the specific binding of the AIs to the corresponding receptors, resulting in a decrease of biofilm formation. BpiB09 is a metagenome-derived NADPdependent reductase and it has been found to be involved in inactivation of the QS signaling molecule OdDHL. Although the AHLs of $P$. aeruginosa are probably not the native substrate of BpiB09, the expression of the enzyme in $P$. aeruginosa results in poor biofilm formation, significantly reduced pyocyanin production and decreased motility (Bijtenhoorn et al., 2011). Another AHL oxidoreductase, Hod, a 2,4-dioxygenase, is capable of catalyzing the conversion of PQS to $\mathrm{N}$-octanoylanthranilic acid and carbon monoxide. Exogenous supplementation of Hod protein into $P$. aeruginosa cultures reduces expression of the PQS biosynthetic enzyme PqsA and the PQS-regulated virulence factors (Pustelny et al., 2009). In short, these QQ enzymes can effectively inhibit bacterial biofilm formation.

Inhibiting the biosynthesis of QS signaling molecules may be a more direct way to interrupt the QS system and inhibit biofilm formation. Some QS inhibitors have been found to inhibit the synthetic activities of AIs synthases. TofI is an AHL synthase identified in the Gram-negative bacterium Burkholderia gluma. The binding site of Tofl can be occupied by an AHL analog and therefore disturb AHL synthesis (Chung et al., 2011). Another example is diketopiperazines that target CepI, an AHL synthase of $B$. cenocepacia, can interfere with the activity of signaling molecule synthases, rendering the bacteria unable to produce biofilms (Buroni et al., 2018).

\section{Targeting the Signaling Molecule Receptors}

The second mechanism of QS inhibiting agents is by targeting the receptors of the QS signaling molecules, thereby inactivating the receptor or competing for the receptor. In most cases, the ligand binding domains of receptors with the native AIs are highly conserved (Khan et al., 2019), which can be competitively or non-competitively bound by most AIs (Table 2). Two classes of 
TABLE 1 | Studies on controlling biofilm by targeting QS signaling molecule.

\begin{tabular}{|c|c|c|c|c|c|}
\hline Source & QS-inhibiting agents & $\begin{array}{l}\text { Chemical } \\
\text { structure }\end{array}$ & Target bacteria & Effects & References \\
\hline Bacillus cereus VT96 & AHL-lactonase AiiA & NA & $\begin{array}{l}\text { P. aeruginosa } \\
\text { V. cholerae } \\
\text { E. cloacae }\end{array}$ & $\begin{array}{l}\text { Degraded AHLs, prevent the biofilm } \\
\text { formation and production of } \\
\text { virulence factors }\end{array}$ & $\begin{array}{l}\text { Augustine et al., 2010; dos } \\
\text { Reis Ponce et al., 2012; } \\
\text { Anandan and Vittal, } 2019\end{array}$ \\
\hline Synthesis & $\begin{array}{l}\text { Molecularly imprinted polymers } \\
\text { (MIPs) }\end{array}$ & & $P$. aeruginosa & $\begin{array}{l}\text { Captured OdDHL, therefor } \\
\text { interrupted QS, and subsequently } \\
\text { inhibit biofilm formation }\end{array}$ & Ma et al., 2018 \\
\hline $\begin{array}{l}\text { Arthrobacter } \\
\text { nitroguajacolicus strain } \\
\text { Rü61a }\end{array}$ & $\begin{array}{l}\text { 3-Hydroxy-2-methyl-4(1H)- } \\
\text { quinolone 2,4-dioxygenase } \\
\text { Hod }\end{array}$ & NA & $P$. aeruginosa & $\begin{array}{l}\text { Catalyzed the conversion of PQS to } \\
\text { N-octanoylanthranilic acid and } \\
\text { carbon monoxide, reduced the } \\
\text { expression of the PQS-regulated } \\
\text { virulence }\end{array}$ & Pustelny et al., 2009 \\
\hline Derivative & Boronic acid derivate SM23 & NA & $P$. aeruginosa & $\begin{array}{l}\text { Decreased 3-oxo-C12-HSL and } \\
\text { C4-HSL and reduced biofilm } \\
\text { formation }\end{array}$ & Peppoloni et al., 2020 \\
\hline Synthesis & Acyl-HSL analog J8-C8 & & B. gluma & $\begin{array}{l}\text { Bound to Tofl, disturbed C8-HSL } \\
\text { synthesis, affected biofilm formation }\end{array}$ & Chung et al., 2011 \\
\hline Synthesis & Diketopiperazines & & B. cenocepacia & $\begin{array}{l}\text { Interfered with the activity of signal } \\
\text { molecule synthase Cepl and } \\
\text { rendered the bacteria unable to } \\
\text { produce biofilm }\end{array}$ & Buroni et al., 2018 \\
\hline Synthesis & $\begin{array}{l}\text { Anti-autoinducer monoclonal } \\
\text { antibody AP4-24H11 }\end{array}$ & NA & S. aureus RN4850 & $\begin{array}{l}\text { Sequestrated the autoinducing } \\
\text { peptide (AIP)-4, inhibited QS and } \\
\text { biofilm formation }\end{array}$ & Park et al., 2007 \\
\hline $\begin{array}{l}\text { Gene from a soil } \\
\text { metagenome }\end{array}$ & $\begin{array}{l}\text { NADP-dependent reductase } \\
\text { BpiB09 }\end{array}$ & NA & P. aeruginosa PAO1 & $\begin{array}{l}\text { Reduced pyocyanin production, } \\
\text { decreased motility, poor biofilm } \\
\text { formation }\end{array}$ & Bijtenhoorn et al., 2011 \\
\hline Synthesis & $\begin{array}{l}\text { 3-(dibromomethylene) } \\
\text { isobenzofuran-1(3H)-one } \\
\text { derivatives }\end{array}$ & & $\begin{array}{l}\text { F. nucleatum } \\
\text { P.gingivalis } \\
\text { T. forsythia }\end{array}$ & $\begin{array}{l}\text { Inhibited biofilm formation through } \\
\text { the inhibition of Al- } 2 \text { activity }\end{array}$ & Park et al., 2017 \\
\hline
\end{tabular}

NA, not available.

QS inhibiting agents, flavonoids and furanones, have been found that can bind to receptors of a variety of pathogenic bacteria (Paczkowski et al., 2017; Proctor et al., 2020). A plant flavonoid, naringenin, competes with the physiological signaling molecule OdDHL by directly binding to the receptor LasR, resulting in inhibition of the production of the QS-regulated virulence factors, pyocyanin and elastase, etc., in P. aeruginosa (HernandoAmado et al., 2020). Also in P. aeruginosa, the receptor LasR can interact with sitagliptin, a drug used for the treatment of diabetes mellitus type 2 , and a minor inhibitory concentration of sitagliptin significantly inhibits biofilm formation (Abbas et al., 2020). In addition, flavonoids can non-competitively bind to the LasR LBD and prevent the protein from binding to DNA, causing repression of certain QS behaviors (Paczkowski et al., 2017). Moreover, some QS inhibiting agents can bind to different receptors at the same time. For example, 3-benzene lactic acid (PLA), a QS inhibiting agent produced by Lactobacillus, antagonistically binds to the receptors RhlR and PqsR with a higher affinity than its cognate ligands BHL and PQS in P. aeruginosa (Chatterjee et al., 2017).

As another class of QS inhibiting agents, furanones, can compete with the native AIs to bind to, and subsequently block, the AHL receptors. They have been demonstrated to significantly decrease virulence factor production and biofilm formation in a range of bacterial species (Proctor et al., 2020). In addition to competing with signaling molecules for receptors, some QS inhibiting agents, such as meta-bromo-thiolactone, can also directly inactivate the receptors to prevent virulence factors expression and biofilm formation (Sully et al., 2014).

\section{Blocking the Signaling Cascade}

The third mechanism of inactivation of QS systems is blocking the signaling cascade by deactivating the downstream response regulators or other regulatory factors. For example, in the AIP system of $S$. aureus, downstream response regulator AgrA is phosphorylated and thereby activated, which is triggered by upstream signaling, and it binds to DNA sequences associated with promoters and upregulates the expression of relevant genes as described above. Inhibiting the response regulators can block the signaling cascade and prevent the formation of a bacterial biofilm (Table 3). Savarin, for example, is a small molecule identified as an $S$. aureus virulence inhibitor, which can specially target AgrA to disrupt agr operon-mediated QS, and hence inhibit biofilm formation (Sully et al., 2014). 
TABLE 2 | Studies on controlling biofilm by targeting QS signaling receptors.

\begin{tabular}{|c|c|c|c|c|c|}
\hline Source & $\begin{array}{l}\text { QS-inhibiting } \\
\text { agents }\end{array}$ & Chemical structure & Target bacteria & Effects & References \\
\hline Lactobacillus & $\begin{array}{l}\text { 3-Phenyllactic acid } \\
\text { (PLA) }\end{array}$ & & $P$. aeruginosa & $\begin{array}{l}\text { Bound to QS receptors RhIR and PqsR } \\
\text { with high affinity, thus inhibited the } \\
\text { expression of virulence factors such as } \\
\text { protease, pyocyanin and rhamnolipids } \\
\text { that are involved in the biofilm formation }\end{array}$ & Chatterjee et al., 2017 \\
\hline Plant & Naringenin & & $P$. aeruginosa & $\begin{array}{l}\text { Competed with OdDHL by directly } \\
\text { binding the QS regulator LasR, inhibited } \\
\text { the production of the QS-regulated } \\
\text { virulence factors, pyocyanin and } \\
\text { elastase }\end{array}$ & $\begin{array}{l}\text { Hernando-Amado et al., } \\
2020\end{array}$ \\
\hline Synthesis & Furanones & & P. aeruginosa & $\begin{array}{l}\text { Competed with the native autoinducers } \\
\text { to bind to the AHL receptors, and } \\
\text { significantly decreased virulence factor } \\
\text { production and biofilm formation }\end{array}$ & Proctor et al., 2020 \\
\hline Synthesis & $\begin{array}{l}\text { Meta-bromo- } \\
\text { thiolactone }\end{array}$ & & P. aeruginosa & $\begin{array}{l}\text { Inhibited receptors LasR and RhIR, } \\
\text { prevented virulence factor expression } \\
\text { and biofilm formation }\end{array}$ & O'Loughlin et al., 2013 \\
\hline Synthesis & $\begin{array}{l}\mathrm{N} \text {-phenyl-4-(3- } \\
\text { phenylthioureido) } \\
\text { benzenesulfonamide }\end{array}$ & & $\begin{array}{l}\text { E. coli(EAEC) } \\
\text { O104:H4 }\end{array}$ & $\begin{array}{l}\text { allosterically modified Al-3 receptor } \\
\text { QseC,impeded virulence expression } \\
\text { and decreased biofilm formation }\end{array}$ & Curtis et al., 2014 \\
\hline
\end{tabular}

TABLE 3 | Studies on controlling biofilm by blocking the signaling cascade.

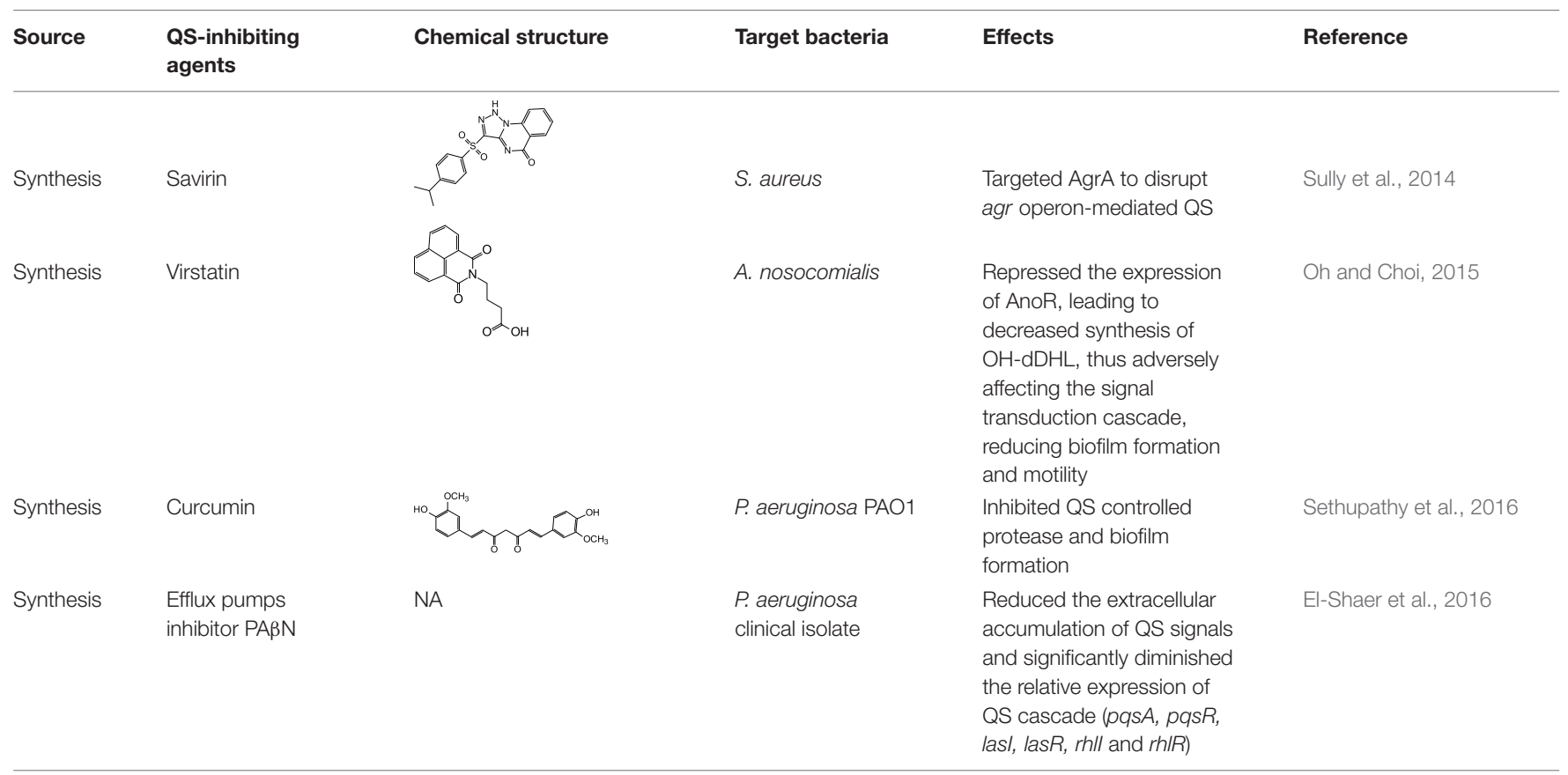

NA, not available. 
In addition to acting on the response regulator, the QS inhibiting agents can also act on other regulatory factors to block signaling cascades. For example, virstatin, a small molecule that acts to prevent the expression of cholerae virulence factors, can repress the expression of $\mathrm{AnoR}$, which is a positive regulator of the LuxI-like synthase AnoI in Acinetobacter nosocomialis, leading to decreased synthesis of $\mathrm{N}$-(3-hydroxy-dodecanoyl)-Lhomoserine lactone (OH-dDHL), thus affecting the signaling cascade, and reducing biofilm formation and motility (Oh and Choi, 2015). Moreover, efflux pumps inhibitor PA $\beta N$ reduces the extracellular accumulation of QS signaling molecules and significantly decreases the relative expression of the QS cascade (pqsA, pqsR, lasI, lasR, rhlI and rhlR) in P. aeruginosa clinical isolates, and this is followed by a reduction of bacterial virulence (El-Shaer et al., 2016).

\section{STRATEGIES OF EXPLOITING NEW QS INHIBITING AGENTS}

In the past two decades, researchers have discovered plenty of QS inhibiting agents that can effectively inhibit biofilm formation in bacteria. The development of new QS inhibiting agents that can effectively replace antibiotics has been a hot topic in the antibacterial research field. With the increasing degree of bacterial resistance to antibiotics, it has become more urgent to develop new QS inhibiting agents that can effectively inhibit biofilm formation. Here, we will introduce four strategies for exploiting new QS inhibitors.

\section{Synthesis of Derivatives of Known QSIs}

Some QS inhibitors are usually structural analogs of AIs, which cause the inactivation of QS by competitively binding to the receptor. Therefore, the synthesis of derivatives of known QSIs that do not alter the core structure may be an effective strategy. Actually, researchers have used natural and chemically synthesized halogenated furanones to successfully synthesize furanone analogs bearing alkyl chains, vinyl bromide keys or aromatic rings, and these analogs can also inhibit biofilm formation (Chang et al., 2019). In addition, C-5 aromatic substituted furanones have been designed and synthesized based on 5-hydroxyl-3,4-halogenated-5H-furan-2-ones, and this compound shows remarkable inhibition of biofilm formation as well as inhibition of virulence factor production in $P$. aeruginosa (Chang et al., 2019). Moreover, a new class of brominated furanones that contained a bicyclic structure were designed and synthesized, and this class of molecules exhibited reduction in the toxicity to mammalian cells, but retained the inhibitory activity toward biofilm formation of bacteria (Yang et al., 2014).

\section{Modification of Existing QQ Enzymes}

The use of quenching enzymes to interfere with QS is an attractive strategy to fight bacterial infections. Intentional modification of QQ enzymes by using protein engineering methods may obtain greater efficiency and stability of quenching enzymes. Thus, this could be an effective strategy for developing new QQ enzymes. MomL is a marine lactonase that can degrade various AHLs.
Two MomL mutants, MomL ${ }^{\mathrm{I} 144 \mathrm{~V}}$ and MomL V149A, exhibit higher activities to block the production of virulence factors of Pectobacterium carotovorum subsp. carotovorum (Pcc) (Wang et al., 2019). PvdQ is an acylase with effective QQ activity in $P$. aeruginosa, but it hardly hydrolyses short-chain AHLs. However, its variant $\mathrm{PvdQ}^{\mathrm{L} \alpha} 146 \mathrm{~W}, \mathrm{~F} \beta 24 Y$ has an altered substrate specificity, and it exhibits high hydrolysis activity toward shorterchain AHLs such as C8-HSL (Koch et al., 2014).

\section{Search for QS Inhibiting Agents in Natural Products}

There are abundant biochemical resources in plants and microorganisms in nature. In recent years, a number of natural products have been found to have potential abilities of inhibition of QS signaling and biofilm formation with the extensive use of computer-aided programs like structure-based virtual screening (SB-VS) and molecular docking bioassays (Ahmed et al., 2019; Borges and Simoes, 2019). Natural plantderived compounds trans-cinnamaldehyde (CA) and salicylic acid (SA) can significantly inhibit the expression of QS-regulated genes involved in virulence, rhamnolipid and reduced biofilm formation in P. aeruginosa (Ahmed et al., 2019). Recently, a novel AHL acylase, MacQ, has been identified from a multidrugresistant bacterium, Acidovorax sp. strain MR-S7, and it was able to degrade a wide variety of AHLs, ranging from C6 to C14 side chains with or without 3-oxo substitutions, thus interfering with the QS system in the bacterial pathogen (Kusada et al., 2017).

\section{Identify Approved Drugs as QS Inhibiting Agents}

The search for QS inhibiting agents from the library of drugs approved for clinical applications may be a promising way to shorten the period of development of an anti-QS drug from discovery to clinical use, since long-term clinical trials are needed to ensure QS inhibiting agents are safe and reliable before they can be used to treat infectious diseases. A typical example of a new therapeutic use of an old drug is niclosamide, which is an FDA approved anti-helminthic drug. Niclosamide has been identified to strongly inhibit the QS response by targeting the las QS system, thereby reducing the expression of las regulon-controlled virulence factors and biofilm formation in $P$. aeruginosa (Imperi et al., 2013). Another FDA approved drug that is used for the treatment of upper respiratory tract infections and for tracheobronchial infections caused by Gram-positive pathogens, clofoctol, has also been found to significantly reduce biofilm formation through inhibiting the pqs QS system in P. aeruginosa (D'Angelo et al., 2018). In addition, albendazole, also an FDA approved clinical drug, has been found to have great potential to act as a QS inhibitor. It quenches the QS by interacting with the hydrophobic amino acid residues of the hydrophobic pocket of CviR and LasR receptors in P. aeruginosa (Singh and Bhatia, 2018). Antibiotics have also shown great potential as QS inhibiting agents. For exsample, aminoglycosides, a commonly used class of antibiotics, exhibit biofilm inhibition by targeting the QS 
regulatory protein LasR in $P$. aeruginosa (Khan et al., 2020a; Khan et al., 2020b).

\section{APPLICATIONS OF QS-INHIBITING AGENTS IN CONTROLLING BACTERIAL BIOFILM FORMATION}

As a new type of antimicrobial agent, QS inhibiting agents have been applied in several fields, such as in medical treatments, food processing and water treatment.

Currently, QS inhibiting agents are widely used in healthrelated fields. $P$. aeruginosa is a major cause of nosocomial infections, especially in the pulmonary infections associated with cystic fibrosis. This organism shows a remarkable capacity to resist antibiotics. Alternative drugs have proven useful against this multiresistant strain. The acylase PvdQ has been identified as an QS inhibiting agent that can irreversibly hydrolyze AHL signaling molecules and has great therapeutic efficacy against pulmonary infections in a mouse model (Utari et al., 2018).

In addition, owing to biofilms being one of the important virulence factors of pathogenic bacteria, infections resulting from the formation of biofilms on medical devices remains a significant clinical problem (Ozcelik et al., 2017). Therefore, a poly(ethylene glycol) (PEG) based multifunctional coating that allows for the covalent incorporation of the synthetic QS inhibitor 5-methylene-1-(prop-2-enoyl)-4-(2-fluorophenyl)dihydropyrrol-2-one (DHP) in a surface can reduce biofilm formation, and the use of this coating can reduce bacterial colonization (Ozcelik et al., 2017). Obviously, this provides a useful way to prevent device-related infections. Moreover, QS inhibiting agents can also be used as antibiotic accelerants for treating bacterial infections. Two cinnamic acid derivatives, 4dimethylaminocinnamic acid (DCA) and 4-methoxycinnamic acid (MCA) as AHL inhibitors, were both found to not only markedly inhibit biofilm formation, but also to enhance the susceptibility of biofilms to tobramycin (Cheng et al., 2020).

In addition to great application prospects in the health-related fields, QS inhibiting agents can also be used in the food industry. Food safety has always been a major concern in the food industry. The formation of biofilms enhances the attachment of bacterial pathogen to the surface of food packaging bags or processing equipment, which increases post-processing contamination and risks to public health due to their persistence and resistance to cleaning and disinfection procedures (Shi and Zhu, 2009). Thus, the extraction of QS inhibiting agents from foodborne materials to solve food contamination problems is a relatively effective approach. The essential oils that were extracted from Murraya koenigii were found to have strong QS inhibitory and anti-biofilm activities that can reduce cell attachment, metabolic activity and EPS production, and in addition, they could delay the decomposition of refrigerated milk caused by psychrophila PSPF19 (Bai and Vittal, 2014). Additional applications of QS inhibiting agents in the food industry are still being explored.

At present, the application of quenching QS in water treatment has also been reported, and it shows great potential and commercial prospects. In wastewater treatment, membrane bioreactor (MBR) is an important technique that combines the activated sludge and membrane filtration processes (Yeon et al., 2009a,b; Lee et al., 2018; Oh and Lee, 2018). However, biofouling, which is mainly caused by the generation of a thickened biofilm layer resulting from bacterial gathering, is a primary problem during the use of MBR (Kose-Mutlu et al., 2019), which seriously affects the efficiency of wastewater treatment. QQ enzymes can be employed to prevent biofouling in MBR. For instance, Kim et al. (2011) immobilized a QQ enzyme (acylase) onto a nano-filtration membrane, and found the acylase-immobilized membrane prohibits the formation of the mushroom-shaped mature biofilm and prevents more than $90 \%$ of the initial flux after $38 \mathrm{~h}$ operation, while the unimmobilized raw membrane dropped to $60 \%$ accompanied by severe biofouling.

There are studies showing that the use of QS inhibiting agents may also be an effective strategy to eradicate biofilm contamination in metalworking fluids (MWFs). Biofilm contamination is a critical problem in MWFs, which not only affects product quality, but also shortens the lifetime of MWFs. Two QS inhibiting agents, patulin and furanone C-30, both reduced biofilm formation in MWF when compared to untreated controls (Ozcan et al., 2019).

\section{CONCLUSION AND FUTURE RESEARCH}

Biofilms can promote bacteria survival in harsh environments. Conventional antibiotics and bactericides cannot penetrate the extracellular matrix of biofilms, resulting in decreased bacterial sensitivity, and therefore, biofilm-related pollution poses serious problems in many fields, including the environment, food, and human diseases. The formation of a biofilm is regulated by the QS system, and thus the use of QS inhibiting agents is a promising strategy to control biofilm formation, and it has been successfully applied in a number of fields.

QS inhibiting agents control biofilm formation generally by targeting QS signaling molecules or their receptors, or downstream regulatory factors. They do not kill bacteria or inhibit the growth of bacteria, and instead they interfere with the expression of virulence factors and inhibit biofilm formation, which puts less pressure on bacterial survival and reduces their drug resistance. This is completely different from the mechanism of antibiotics killing bacteria.

In recent years, many natural or synthetic QS inhibiting agents that effectively reduce biofilm formation have been exploited. However, there are still several problems to be overcome: (1) the mechanisms of some QS inhibiting agents in controlling biofilms are still unclear; (2) some known QS inhibiting agents are cytotoxic or unstable; (3) the anti-biofilm effect is not broad enough, only being effective against one or two bacterial species; (4) what are the best conditions for QS inhibiting agents in controlling biofilms? (5) whether QS inhibiting agents have an impact on beneficial bacteria in the environment. These problems all need to be overcome to develop more efficient, less toxic QS inhibiting agents that will provide great value in the future for biofilm prevention and treatment. 


\section{AUTHOR CONTRIBUTIONS}

JP and LZ provided the general concept and designed the manuscript and revised and approved the manuscript. LZ, YG, $\mathrm{YZ}$, and $\mathrm{XZ}$ wrote the manuscript. LZ contributed to create the figure. All authors contributed to the article and approved the submitted version.

\section{REFERENCES}

Abbas, H. A., Shaldam, M. A., and Eldamasi, D. (2020). Curtailing quorum sensing in Pseudomonas aeruginosa by Sitagliptin. Curr. Microbiol. 77, 1051-1060. doi: 10.1007/s00284-020-01909-1904

Ahmed, S., Rudden, M., Smyth, T. J., Dooley, J. S. G., Marchant, R., and Banat, I. M. (2019). Natural quorum sensing inhibitors effectively downregulate gene expression of Pseudomonas aeruginosa virulence factors. Appl. Microbiol. Biotechnol. 103, 3521-3535. doi: 10.1007/s00253-019-09618-9610

Allesen-Holm, M., Barken, K. B., Yang, L., Klausen, M., Webb, J. S., Kjelleberg, S., et al. (2006). A characterization of DNA release in Pseudomonas aeruginosa cultures and biofilms. Mol. Microbiol. 59, 1114-1128. doi: 10.1111/j.1365-2958. 2005.05008.x

Anandan, K., and Vittal, R. R. (2019). Quorum quenching activity of AiiA lactonase KMMI17 from endophytic Bacillus thuringiensis KMCL07 on AHL- mediated pathogenic phenotype in Pseudomonas aeruginosa. Microb. Pathog. 132, 230242. doi: 10.1016/j.micpath.2019.05.015

Anderson, J. K., Huang, J. Y., Wreden, C., Sweeney, E. G., Goers, J., Remington, S. J., et al. (2015). Chemorepulsion from the Quorum Signal Autoinducer2 Promotes Helicobacter pylori Biofilm Dispersal. mBio 6:e00379-15. doi: 10. 1128/mBio.00379-315

Aswathanarayan, J. B., and Vittal, R. R. (2016). Effect of small chain N acyl homoserine lactone quorum sensing signals on biofilms of food-borne pathogens. J. Food Sci. Technol. 53, 3609-3614. doi: 10.1007/s13197-016-23462341

Augustine, N., Kumar, P., and Thomas, S. (2010). Inhibition of Vibrio cholerae biofilm by AiiA enzyme produced from Bacillus spp. Arch. Microbiol. 192, 1019-1022. doi: 10.1007/s00203-010-0633-631

Bai, A. J., and Vittal, R. R. (2014). Quorum sensing inhibitory and anti-biofilm activity of essential oils and their in vivo efficacy in food systems. Food Biotechnol. 28, 269-292. doi: 10.1080/08905436.2014.932287

Bassler, B. L., Greenberg, E. P., and Stevens, A. M. (1997). Cross-species induction of luminescence in the quorum-sensing bacterium Vibrio harveyi. J. Bacteriol. 179, 4043-4045. doi: 10.1128/jb.179.12.4043-4045.1997

Bijtenhoorn, P., Mayerhofer, H., Muller-Dieckmann, J., Utpatel, C., Schipper, C., Hornung, C., et al. (2011). A novel metagenomic short-chain dehydrogenase/reductase attenuates Pseudomonas aeruginosa biofilm formation and virulence on Caenorhabditis elegans. PLoS One 6:e26278. doi: 10.1371/journal.pone.0026278

Borges, A., and Simoes, M. (2019). Quorum sensing inhibition by marine bacteria. Mar. Drugs 17:427. doi: 10.3390/md17070427

Branda, S. S., Vik, S., Friedman, L., and Kolter, R. (2005). Biofilms: the matrix revisited. Trends Microbiol. 13, 20-26. doi: 10.1016/j.tim.2004.11.006

Buroni, S., Scoffone, V. C., Fumagalli, M., Makarov, V., Cagnone, M., Trespidi, G., et al. (2018). Investigating the mechanism of action of diketopiperazines inhibitors of the Burkholderia cenocepacia quorum sensing synthase cepi: a sitedirected mutagenesis study. Front. Pharmacol. 9:836. doi: 10.3389/fphar.2018. 00836

Chang, Y. Q., Wang, P. C., Ma, H. M., Chen, S. Y., Fu, Y. H., Liu, Y. Y., et al. (2019). Design, synthesis and evaluation of halogenated furanone derivatives as quorum sensing inhibitors in Pseudomonas aeruginosa. Eur. J. Pharm. Sci. 140:105058. doi: 10.1016/j.ejps.2019.105058

Chatterjee, M., D’Morris, S., Paul, V., Warrier, S., Vasudevan, A. K., Vanuopadath, M., et al. (2017). Mechanistic understanding of Phenyllactic acid mediated inhibition of quorum sensing and biofilm development in Pseudomonas aeruginosa. Appl. Microbiol. Biotechnol. 101, 8223-8236. doi: 10.1007/s00253017-8546-8544

\section{FUNDING}

This work was supported by grants from the National Natural Science Foundation of China (31701572), the National Natural Science Foundation of China (31770141), and the 521 Talent Program of Zhejiang Sci-Tech University, China, to JP.

Cheng, W. J., Zhou, J. W., Zhang, P. P., Luo, H. Z., Tang, S., Li, J. J., et al. (2020). Quorum sensing inhibition and tobramycin acceleration in Chromobacterium violaceum by two natural cinnamic acid derivatives. Appl. Microbiol. Biotechnol. 104, 5025-5037. doi: 10.1007/s00253-020-10593-10590

Chung, J., Goo, E., Yu, S., Choi, O., Lee, J., Kim, J., et al. (2011). Small-molecule inhibitor binding to an N-acyl-homoserine lactone synthase. Proc. Natl. Acad. Sci. U.S.A. 108, 12089-12094. doi: 10.1073/pnas.1103165108

Costerton, J. W., Lewandowski, Z., DeBeer, D., Caldwell, D., Korber, D., and James, G. (1994). Biofilms, the customized microniche. J. Bacteriol. 176, 2137-2142. doi: 10.1128/jb.176.8.2137-2142.1994

Curtis, M. M., Russell, R., Moreira, C. G., Adebesin, A. M., Wang, C. G., Williams, N. S., et al. (2014). QseC inhibitors as an antivirulence approach for gramnegative pathogens. mBio 5:e2165-14. doi: 10.1128/mBio.02165-2114

D’Angelo, F., Baldelli, V., Halliday, N., Pantalone, P., Polticelli, F., Fiscarelli, E., et al. (2018). Identification of FDA-Approved Drugs as Antivirulence Agents Targeting the pqs Quorum-Sensing System of Pseudomonas aeruginosa. Antimicrob. Agents Chemother. 62:e1296-18.

Daniels, R., Vanderleyden, J., and Michiels, J. (2004). Quorum sensing and swarming migration in bacteria. FEMS Microbiol. Rev. 28, 261-289. doi: 10. 1016/j.femsre.2003.09.004

Ding, X., Yin, B., Qian, L., Zeng, Z., Yang, Z., Li, H., et al. (2011). Screening for novel quorum-sensing inhibitors to interfere with the formation of Pseudomonas aeruginosa biofilm. J. Med. Microbiol. 60(Pt 12), 1827-1834. doi: 10.1099/jmm. $0.024166-24160$

Donlan, R. M. (2002). Biofilms: microbial life on surfaces. Emerg. Infect. Dis. 8, 881-890. doi: 10.3201/eid0809.020063

dos Reis Ponce, A., Martins, M. L., de Araujo, E. F., Mantovani, H. C., and Vanetti, M. C. (2012). AiiA quorum-sensing quenching controls proteolytic activity and biofilm formation by Enterobacter cloacae. Curr. Microbiol. 65, 758-763. doi: 10.1007/s00284-012-0226-220

Dusane, D. H., Zinjarde, S. S., Venugopalan, V. P., McLean, R. J., Weber, M. M., and Rahman, P. K. (2010). Quorum sensing: implications on rhamnolipid biosurfactant production. Biotechnol. Genet. Eng. Rev. 27, 159-184. doi: 10. 1080/02648725.2010.10648149

El-Shaer, S., Shaaban, M., Barwa, R., and Hassan, R. (2016). Control of quorum sensing and virulence factors of Pseudomonas aeruginosa using phenylalanine arginyl beta-naphthylamide. J. Med. Microbiol. 65, 1194-1204. doi: 10.1099/ jmm.0.000327

Engebrecht, J., Nealson, K., and Silverman, M. (1983). Bacterial bioluminescence: isolation and genetic analysis of functions from Vibrio fischeri. Cell 32, 773-781. doi: 10.1016/0092-8674(83)90063-90066

Engebrecht, J., and Silverman, M. (1984). Identification of genes and gene products necessary for bacterial bioluminescence. Proc. Natl. Acad. Sci. U.S.A. 81, 4154 4158. doi: 10.1073/pnas.81.13.4154

Guo, M., Fang, Z., Sun, L., Sun, D., Wang, Y., Li, C., et al. (2018). Regulation of thermostable direct hemolysin and biofilm formation of Vibrio parahaemolyticus by Quorum-Sensing Genes luxM and luxS. Curr. Microbiol. 75, 1190-1197. doi: 10.1007/s00284-018-1508-y

Hammer, B. K., and Bassler, B. L. (2003). Quorum sensing controls biofilm formation in Vibrio cholerae. Mol. Microbiol. 50, 101-104. doi: 10.1046/j.13652958.2003.03688.x

Hammer, B. K., and Bassler, B. L. (2007). Regulatory small RNAs circumvent the conventional quorum sensing pathway in pandemic Vibrio cholerae. Proc. Natl. Acad. Sci. U.S.A. 104, 11145-11149. doi: 10.1073/pnas.070386 0104

Hernando-Amado, S., Alcalde-Rico, M., Gil-Gil, T., Valverde, J. R., and Martinez, J. L. (2020). Naringenin Inhibition of the Pseudomonas aeruginosa Quorum 
Sensing Response Is Based on Its Time-Dependent Competition With N-(3Oxo-dodecanoyl)-L-homoserine Lactone for LasR Binding. Front. Mol. Biosci. 7:25. doi: $10.3389 /$ fmolb. 2020.00025

Hoiby, N., Bjarnsholt, T., Givskov, M., Molin, S., and Ciofu, O. (2010). Antibiotic resistance of bacterial biofilms. Int. J. Antimicrob. Agents 35, 322-332. doi: 10.1016/j.ijantimicag.2009.12.011

Imperi, F., Massai, F., Ramachandran Pillai, C., Longo, F., Zennaro, E., Rampioni, G., et al. (2013). New life for an old drug: the anthelmintic drug niclosamide inhibits Pseudomonas aeruginosa quorum sensing. Antimicrob. Agents Chemother. 57, 996-1005. doi: 10.1128/AAC.01952-1912

Irie, Y., and Parsek, M. R. (2008). Quorum sensing and microbial biofilms. Curr. Top. Microbiol. Immunol. 322, 67-84. doi: 10.1007/978-3-540-75418-3_4

Khan, F., Javaid, A., and Kim, Y. M. (2019). Functional diversity of quorum sensing receptors in pathogenic bacteria: interspecies, intraspecies and interkingdom level. Curr. Drug Targets 20, 655-667. doi: 10.2174/ 1389450120666181123123333

Khan, F., Lee, J. W., Javaid, A., Park, S. K., and Kim, Y. M. (2020a). Inhibition of biofilm and virulence properties of Pseudomonas aeruginosa by sub-inhibitory concentrations of aminoglycosides. Microb. Pathog. 146:104249. doi: 10.1016/j. micpath.2020.104249

Khan, F., Pham, D. T. N., and Kim, Y. M. (2020b). Alternative strategies for the application of aminoglycoside antibiotics against the biofilm-forming human pathogenic bacteria. Appl. Microbiol. Biotechnol. 104, 1955-1976. doi: 10.1007/ s00253-020-10360-10361

Khan, F., Pham, D. T. N., Oloketuyi, S. F., and Kim, Y. M. (2020c). Regulation and controlling the motility properties of Pseudomonas aeruginosa. Appl. Microbiol. Biotechnol. 104, 33-49. doi: 10.1007/s00253-019-10201-w

Kim, C. S., Gatsios, A., Cuesta, S., Lam, Y. C., Wei, Z., Chen, H., et al. (2020). Characterization of autoinducer-3 structure and biosynthesis in E. coli. ACS Cent. Sci. 6, 197-206. doi: 10.1021/acscentsci.9b01076

Kim, J. H., Choi, D. C., Yeon, K. M., Kim, S. R., and Lee, C. H. (2011). Enzymeimmobilized nanofiltration membrane to mitigate biofouling based on quorum quenching. Environ. Sci. Technol. 45, 1601-1607. doi: 10.1021/es103483j

Kleerebezem, M., Quadri, L. E., Kuipers, O. P., and de Vos, W. M. (1997). Quorum sensing by peptide pheromones and two-component signal-transduction systems in Gram-positive bacteria. Mol. Microbiol. 24, 895-904. doi: 10.1046/ j.1365-2958.1997.4251782.x

Koch, G., Nadal-Jimenez, P., Reis, C. R., Muntendam, R., Bokhove, M., Melillo, E., et al. (2014). Reducing virulence of the human pathogen Burkholderia by altering the substrate specificity of the quorum-quenching acylase PvdQ. Proc. Natl. Acad. Sci. U.S.A. 111, 1568-1573. doi: 10.1073/pnas.1311263111

Kose-Mutlu, B., Ergon-Can, T., Koyuncu, I., and Lee, C. H. (2019). Quorum quenching for effective control of biofouling in membrane bioreactor: a comprehensive review of approaches, applications, and challenges. Environ. Eng. Res. 24, 543-558. doi: 10.4491/eer.2018.380

Kusada, H., Tamaki, H., Kamagata, Y., Hanada, S., and Kimura, N. (2017). A Novel Quorum-Quenching N-Acylhomoserine lactone acylase from Acidovorax sp. Strain MR-S7 Mediates Antibiotic Resistance. Appl. Environ. Microbiol. 83:e00080-17. doi: 10.1128/AEM.00080-17

Lee, J., and Zhang, L. (2015). The hierarchy quorum sensing network in Pseudomonas aeruginosa. Protein Cell 6, 26-41. doi: 10.1007/s13238-014-0100$\mathrm{x}$

Lee, K., Yu, H. R., Zhang, X. L., and Choo, K. H. (2018). Quorum sensing and quenching in membrane bioreactors: opportunities and challenges for biofouling control. Bioresour. Technol. 270, 656-668. doi: 10.1016/j.biortech. 2018.09.019

Lewis, K. (2001). Riddle of biofilm resistance. Antimicrob. Agents Chemother. 45, 999-1007. doi: 10.1128/AAC.45.4.999-1007.2001

Li, H., Li, X., Wang, Z., Fu, Y., Ai, Q., Dong, Y., et al. (2015). Autoinducer2 regulates Pseudomonas aeruginosa $\mathrm{PAO} 1$ biofilm formation and virulence production in a dose-dependent manner. BMC Microbiol. 15:192. doi: 10.1186/ s12866-015-0529-y

Li, J., Attila, C., Wang, L., Wood, T. K., Valdes, J. J., and Bentley, W. E. (2007). Quorum sensing in Escherichia coli is signaled by AI-2/LsrR: effects on small RNA and Biofilm architecture. J. Bacteriol. 189, 6011-6020. doi: 10.1128/Jb. 00014-17

Ma, L., Feng, S., Fuente-Nunez, C., Hancock, R. E. W., and Lu, X. (2018). Development of molecularly imprinted polymers to block quorum sensing and inhibit bacterial biofilm formation. ACS Appl. Mater. Interfaces 10, 1845018457. doi: 10.1021 /acsami.8b01584

Ma, R., Qiu, S., Jiang, Q., Sun, H., Xue, T., Cai, G., et al. (2017). AI-2 quorum sensing negatively regulates rbf expression and biofilm formation in Staphylococcus aureus. Int. J. Med. Microbiol. 307, 257-267. doi: 10.1016/j.ijmm. 2017.03.003

Nealson, K. H., and Hastings, J. W. (1979). Bacterial bioluminescence: its control and ecological significance. Microbiol. Rev. 43, 496-518. doi: 10.1128/mmbr.43. 4.496-518.1979

Oh, H. S., and Lee, C. H. (2018). Origin and evolution of quorum quenching technology for biofouling control in MBRs for wastewater treatment. J. Membr. Sci. 554, 331-345. doi: 10.1016/j.memsci.2018.03.019

Oh, M. H., and Choi, C. H. (2015). Role of LuxIR Homologue AnoIR in Acinetobacter nosocomialis and the effect of virstatin on the expression of anoR Gene. J. Microbiol. Biotechnol. 25, 1390-1400. doi: 10.4014/jmb.1504.04069

O’Loughlin, C. T., Miller, L. C., Siryaporn, A., Drescher, K., Semmelhack, M. F., and Bassler, B. L. (2013). A quorum-sensing inhibitor blocks Pseudomonas aeruginosa virulence and biofilm formation. Proc. Natl. Acad. Sci. U.S.A. 110, 17981-17986. doi: 10.1073/pnas.1316981110

Ozcan, S. S., Dieser, M., Parker, A. E., Balasubramanian, N., and Foreman, C. M. (2019). Quorum sensing inhibition as a promising method to control biofilm growth in metalworking fluids. J. Indus. Microbiol. Biotechnol. 46, 1103-1111. doi: 10.1007/s10295-019-02181-7

Ozcelik, B., Ho, K. K. K., Glattauer, V., Willcox, M., Kumar, N., and Thissen, H. (2017). Poly(ethylene glycol)-Based Coatings Combining Low-Biofouling and Quorum-Sensing Inhibiting Properties to Reduce Bacterial Colonization. ACS Biomater. Sci. Eng. 3, 78-87. doi: 10.1021/acsbiomaterials.6b00579

Paczkowski, J. E., Mukherjee, S., McCready, A. R., Cong, J. P., Aquino, C. J., Kim, H., et al. (2017). Flavonoids suppress Pseudomonas aeruginosa virulence through allosteric inhibition of quorum-sensing receptors. J. Biol. Chem. 292, 4064-4076.

Painter, K. L., Krishna, A., Wigneshweraraj, S., and Edwards, A. M. (2014). What role does the quorum-sensing accessory gene regulator system play during Staphylococcus aureus bacteremia? Trends Microbiol. 22, 676-685. doi: 10.1016/ j.tim.2014.09.002

Park, J., Jagasia, R., Kaufmann, G. F., Mathison, J. C., Ruiz, D. I., Moss, J. A., et al. (2007). Infection control by antibody disruption of bacterial quorum sensing signaling. Chem. Biol. 14, 1119-1127. doi: 10.1016/j.chembiol.2007.08.013

Park, J. S., Ryu, E. J., Li, L. Z., Choi, B. K., and Kim, B. M. (2017). New bicyclic brominated furanones as potent autoinducer-2 quorum-sensing inhibitors against bacterial biofilm formation. Eur. J. Med. Chem. 137, 76-87. doi: 10.1016/ j.ejmech.2017.05.037

Peppoloni, S., Pericolini, E., Colombari, B., Pinetti, D., Cermelli, C., Fini, F., et al. (2020). The beta-lactamase inhibitor boronic acid derivative SM23 as a new anti-Pseudomonas aeruginosa Biofilm. Front. Microbiol. 11:35. doi: 10.3389/ fmicb.2020.00035

Proctor, C. R., McCarron, P. A., and Ternan, N. G. (2020). Furanone quorumsensing inhibitors with potential as novel therapeutics against Pseudomonas aeruginosa. J. Med. Microbiol. 69, 195-206. doi: 10.1099/jmm.0.001144

Pustelny, C., Albers, A., Buldt-Karentzopoulos, K., Parschat, K., Chhabra, S. R., Camara, M., et al. (2009). Dioxygenase-mediated quenching of quinolonedependent quorum sensing in Pseudomonas aeruginosa. Chem. Biol. 16, 12591267. doi: 10.1016/j.chembiol.2009.11.013

Sethupathy, S., Prasath, K. G., Ananthi, S., Mahalingam, S., Balan, S. Y., and Pandian, S. K. (2016). Proteomic analysis reveals modulation of iron homeostasis and oxidative stress response in Pseudomonas aeruginosa PAO1 by curcumin inhibiting quorum sensing regulated virulence factors and biofilm production. J. Proteomics 145, 112-126. doi: 10.1016/j.jprot.2016.04.019

Shi, X. M., and Zhu, X. N. (2009). Biofilm formation and food safety in food industries. Trends Food Sci. Technol. 20, 407-413. doi: 10.1016/j.tifs.2009.01. 054

Singh, S., and Bhatia, S. (2018). In silico identification of albendazole as a quorum sensing inhibitor and its in vitro verification using CviR and LasB receptors based assay systems. Bioimpacts 8, 201-209. doi: 10.15171/bi.2018.23

Sio, C. F., Otten, L. G., Cool, R. H., Diggle, S. P., Braun, P. G., Bos, R., et al. (2006). Quorum quenching by an N-acyl-homoserine lactone acylase from Pseudomonas aeruginosa PAO1. Infect. Immun. 74, 1673-1682. doi: 10.1128/ IAI.74.3.1673-1682.2006 
Slock, J., VanRiet, D., Kolibachuk, D., and Greenberg, E. P. (1990). Critical regions of the Vibrio fischeri luxR protein defined by mutational analysis. J. Bacteriol. 172, 3974-3979. doi: 10.1128/jb.172.7.3974-3979.1990

Solano, C., Echeverz, M., and Lasa, I. (2014). Biofilm dispersion and quorum sensing. Curr. Opin. Microbiol. 18, 96-104. doi: 10.1016/j.mib.2014.02.008

Sperandio, V., Torres, A. G., Jarvis, B., Nataro, J. P., and Kaper, J. B. (2003). Bacteria-host communication: the language of hormones. Proc. Natl. Acad. Sci. U.S.A. 100, 8951-8956. doi: 10.1073/pnas.1537100100

Sperandio, V., Torres, A. G., and Kaper, J. B. (2002). Quorum sensing Escherichia coli regulators $\mathrm{B}$ and $\mathrm{C}(\mathrm{QseBC})$ : a novel two-component regulatory system involved in the regulation of flagella and motility by quorum sensing in E. coli. Mol. Microbiol. 43, 809-821. doi: 10.1046/j.1365-2958.2002.02803.x

Stevens, A. M., Dolan, K. M., and Greenberg, E. P. (1994). Synergistic binding of the Vibrio fischeri LuxR transcriptional activator domain and RNA polymerase to the lux promoter region. Proc. Natl. Acad. Sci. U.S.A. 91, 12619-12623. doi: $10.1073 /$ pnas.91.26.12619

Stewart, P. S. (2002). Mechanisms of antibiotic resistance in bacterial biofilms. Int. J. Med. Microbiol. 292, 107-113. doi: 10.1078/1438-4221-4196

Sturme, M. H., Kleerebezem, M., Nakayama, J., Akkermans, A. D., Vaugha, E. E., and de Vos, W. M. (2002). Cell to cell communication by autoinducing peptides in gram-positive bacteria. Antonie Van Leeuwenhoek 81, 233-243. doi: 10.1023/ a:1020522919555

Sully, E. K., Malachowa, N., Elmore, B. O., Alexander, S. M., Femling, J. K., Gray, B. M., et al. (2014). Selective chemical inhibition of agr quorum sensing in Staphylococcus aureus promotes host defense with minimal impact on resistance. PLoS Pathog. 10:e1004174. doi: 10.1371/journal.ppat.1004174

Surette, M. G., and Bassler, B. L. (1998). Quorum sensing in Escherichia coli and Salmonella typhimurium. Proc. Natl. Acad. Sci. U.S.A. 95, 7046-7050. doi: 10. 1073/pnas.95.12.7046

Sutherland, I. W. (2001). The biofilm matrix-an immobilized but dynamic microbial environment. Trends Microbiol. 9, 222-227. doi: 10.1016/s0966842x(01)02012-2011

Utari, P. D., Setroikromo, R., Melgert, B. N., and Quax, W. J. (2018). PvdQ quorum quenching acylase attenuates Pseudomonas aeruginosa virulence in a mouse model of pulmonary infection. Front. Cell Infect. Microbiol. 8:119. doi: $10.3389 /$ fcimb.2018.00119

Vinothkannan, R., Tamizh, M. M., Raj, C. D., and Princy, S. A. (2018). Fructose furoic acid ester: an effective quorum sensing inhibitor against uropathogenic Escherichia coli. Bioorganic Chem. 79, 310-318. doi: 10.1016/j.bioorg.2018.05. 009

Vuong, C., Durr, M., Carmody, A. B., Peschel, A., Klebanoff, S. J., and Otto, M. (2004). Regulated expression of pathogen-associated molecular pattern molecules in Staphylococcus epidermidis: quorum-sensing determines proinflammatory capacity and production of phenol-soluble modulins. Cell Microbiol. 6, 753-759. doi: 10.1111/j.1462-5822.2004.00401.x

Vuong, C., Saenz, H. L., Gotz, F., and Otto, M. (2000). Impact of the agr quorumsensing system on adherence to polystyrene in Staphylococcus aureus. J. Infect. Dis. 182, 1688-1693. doi: 10.1086/317606

Wade, D. S., Calfee, M. W., Rocha, E. R., Ling, E. A., Engstrom, E., Coleman, J. P., et al. (2005). Regulation of Pseudomonas quinolone signal synthesis in Pseudomonas aeruginosa. J. Bacteriol. 187, 4372-4380. doi: 10.1128/JB.187.13. 4372-4380.2005
Walters, M., and Sperandio, V. (2006). Quorum sensing in Escherichia coli and Salmonella. Int. J. Med. Microbiol. 296, 125-131. doi: 10.1016/j.ijmm.2006.01. 041

Wang, J., Lin, J., Zhang, Y., Zhang, J., Feng, T., Li, H., et al. (2019). Activity improvement and vital amino acid identification on the marine-derived quorum quenching enzyme moml by protein engineering. Mar. Drugs 17:300. doi: $10.3390 / \mathrm{md} 17050300$

Wang, Y., Zhang, W., Wu, Z. F., Zhu, X. L., and Lu, C. P. (2011). Functional analysis of luxS in Streptococcus suis reveals a key role in biofilm formation and virulence. Vet. Microbiol. 152, 151-160. doi: 10.1016/j.vetmic.2011.04.029

Waters, C. M., and Bassler, B. L. (2005). Quorum sensing: cell-to-cell communication in bacteria. Annu. Rev. Cell Dev. Biol. 21, 319-346. doi: 10. 1146/annurev.cellbio.21.012704.131001

Wilder, C. N., Diggle, S. P., and Schuster, M. (2011). Cooperation and cheating in Pseudomonas aeruginosa: the roles of the las, rhl and pqs quorum-sensing systems. ISME J. 5, 1332-1343. doi: 10.1038/ismej.2011.13

Williams, P. (2007). Quorum sensing, communication and cross-kingdom signalling in the bacterial world. Microbiology 153(Pt 12), 3923-3938. doi: 10 . 1099/mic.0.2007/012856-12850

Winzer, K., Falconer, C., Garber, N. C., Diggle, S. P., Camara, M., and Williams, P. (2000). The Pseudomonas aeruginosa lectins PA-IL and PA-IIL are controlled by quorum sensing and by RpoS. J. Bacteriol. 182, 6401-6411. doi: 10.1128/jb.182. 22.6401-6411.2000

Yang, S. J., Abdel-Razek, O. A., Cheng, F., Bandyopadhyay, D., Shetye, G. S., Wang, G. R., et al. (2014). Bicyclic brominated furanones: a new class of quorum sensing modulators that inhibit bacterial biofilm formation. Bioorgan. Med. Chem. 22, 1313-1317. doi: 10.1016/j.bmc.2014.01.004

Yang, Y., Li, W., Hou, B., and Zhang, C. (2018). Quorum sensing LuxS/autoinducer-2 inhibits Enterococcus faecalis biofilm formation ability. J. Appl. Oral. Sci. 26:e20170566. doi: 10.1590/1678-7757-2017-2566

Yarwood, J. M., Bartels, D. J., Volper, E. M., and Greenberg, E. P. (2004). Quorum sensing in Staphylococcus aureus biofilms. J. Bacteriol. 186, 1838-1850. doi: 10.1128/jb.186.6.1838-1850.2004

Yeon, K. M., Cheong, W. S., Oh, H. S., Lee, W. N., Hwang, B. K., Lee, C. H., et al. (2009a). Quorum sensing: a new biofouling control paradigm in a membrane bioreactor for advanced wastewater treatment. Environ. Sci. Technol. 43, 380-385. doi: 10.1021/es8019275

Yeon, K. M., Lee, C. H., and Kim, J. (2009b). Magnetic enzyme carrier for effective biofouling control in the membrane bioreactor based on enzymatic quorum quenching. Environ. Sci. Technol. 43, 7403-7409. doi: 10.1021/es90 $1323 \mathrm{k}$

Conflict of Interest: The authors declare that the research was conducted in the absence of any commercial or financial relationships that could be construed as a potential conflict of interest.

Copyright (c) 2020 Zhou, Zhang, Ge, Zhu and Pan. This is an open-access article distributed under the terms of the Creative Commons Attribution License (CC BY). The use, distribution or reproduction in other forums is permitted, provided the original author(s) and the copyright owner(s) are credited and that the original publication in this journal is cited, in accordance with accepted academic practice. No use, distribution or reproduction is permitted which does not comply with these terms. 